\title{
Effet du mode d'élevage sur la prolificité des truies de race locale du Bénin et la viabilité de leurs porcelets, de la naissance au sevrage
}

\author{
G. B. KOUTINHOUIN ${ }^{1}$, A. K. I. YOUSSAO ${ }^{1}$, S. S. TOLEBA ${ }^{2}$, T. M. KPODEKON ${ }^{1}$, \\ G. S. AHOUNOU ${ }^{1}$, A. G. BONOU ${ }^{1}$ et J. BESSANVI ${ }^{1}$ \\ ${ }^{1}$ Université d'Abomey-Calavi, Ecole Polytechnique d'Abomey-Calavi, Département de Production Animale, \\ 01 B.P 2009, Cotonou, Bénin. \\ ${ }^{2}$ Université d'Abomey-Calavi, Faculté des Sciences Agronomiques, Département de Production Animale, \\ 01 B.P. 526, Cotonou, Bénin. \\ *Auteur correspondant, E-mail : issaka.youssao@epac.uac.bj; Tél. : $0022995285988 / 97912074$
}

\section{RESUME}

L'effet du mode d'élevage sur la prolificité des truies de race locale et la viabilité de leurs porcelets, a été étudié dans 4 localités. Le lot expérimental composé de 30 truies a été suivi sur le plan sanitaire et a reçu un complément d'aliment du dernier mois de la gestation au sevrage des porcelets, tandis que les animaux du lot témoin composés de 36 truies, étaient élevés suivant le mode d'élevage traditionnel classique. La taille de la portée des truies locales du lot témoin a été plus faible $(\mathrm{P}<0,001)$ que celle des truies expérimentales à la naissance et au sevrage. Il a été observé plus de porcelets nés vivants par portée dans le lot expérimental $(8,3)$ que dans le lot témoin $(4,89)$. Le taux de mortinatalité des porcelets du lot témoin $(12,5 \%)$ a été le double de celui du lot expérimental (5,68\%). De la naissance au sevrage, le poids à âge-type des porcelets expérimentaux a été significativement plus élevé par rapport à ceux des témoins $(\mathrm{P}<0,01)$. L'amélioration du mode d'élevage des truies gestantes augmente la taille et le poids de la portée à la naissance et au sevrage, et réduit le taux de mortinatalité.

(c) 2009 International Formulae Group. All rights reserved.

Mots clés: Porc, portée, prolificité, mortalité, poids, croissance, mortinatalité.

\section{INTRODUCTION}

Dans les pays de la sous région ouest africaine en général et au Bénin en particulier, la production de viandes est en dessous des besoins exprimés par les consommateurs et ce déficit est comblé par des importations qui ne cessent d'augmenter d'année en année. En 10 ans, le volume des importations de viande est passé de 16608 tonnes métriques en 1993 à 86535 tonnes métriques en 2003 (FAO, 2005). Il est alors urgent pour le Bénin, à l'instar des autres pays de la sous région, d'intensifier les productions agricoles en général et la production animale en particulier. Le développement de la production des animaux à cycle court (porcs, volailles, lapins et autres) pourrait apporter une solution substantielle au problème d'autosuffisance alimentaire. Au Bénin, la population porcine locale est caractérisée par des performances de croissance et une productivité numérique faibles mais elle possède par contre une bonne rusticité contre les maladies. Elle est mise en reproduction à six mois avec une moyenne de 6 à 7 porcelets par portée (Bonou, 2006; Youssao et al., 2009), avec des taux de mortinatalité et de mortalité respectifs de $33 \%$ et $21,9 \%$ (Attodjinou et Dotcho, 2006). Dans les élevages traditionnels constitués exclusivement de porcs locaux, les performances sont en général médiocres par rapport aux races exotiques. En effet, la 
productivité des races locales béninoises est influencée par le système d'élevage (Honkpehedji, 2005). Dans ce contexte, comment parvenir à améliorer quelque peu la productivité des porcs locaux ?

Le but de cette étude est d'évaluer l'effet du mode de conduite des truies gestantes sur la taille et le poids de la portée des truies d'une part, la viabilité et la croissance des porcelets de la naissance au sevrage d'autre part.

\section{MATERIEL ET METHODES}

Site d'étude

L'expérimentation a été réalisée en zone tropicale Subhumide du Bénin, dans le Département de l'Atlantique. Les sites retenus dans le cadre de cette étude sont: Togba, Ouèdo, Hêvié et Zinvié (Figure 1).

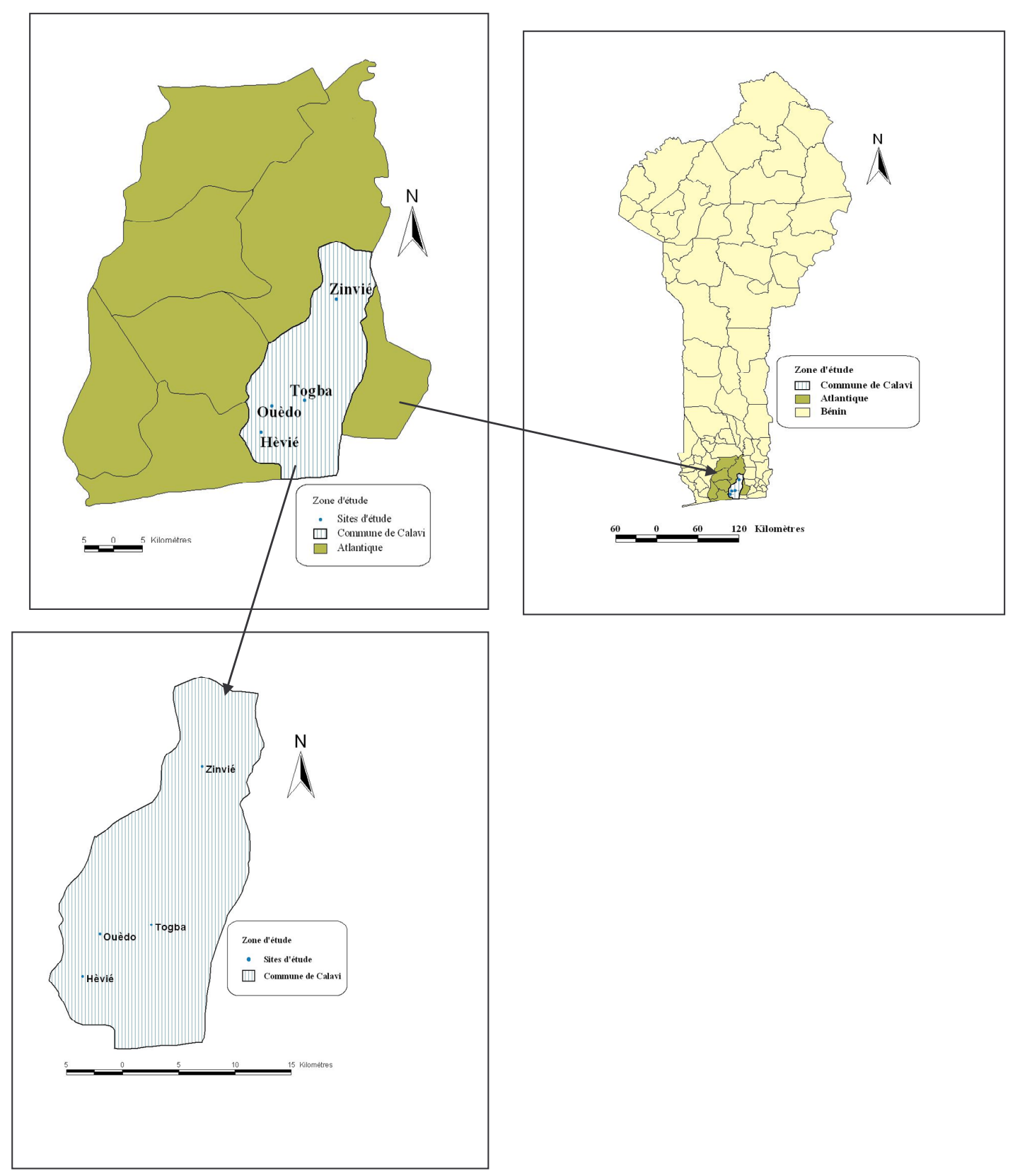

Figure 1 : Zone d'étude 
Ce Département bénéficie d'un climat subéquatorial, caractérisé par deux saisons de pluies : la grande (avril à juillet) et la petite (septembre à novembre), intercalées par deux saisons sèches. La pluviosité moyenne avoisine $1200 \mathrm{~mm}$ par an. Les températures moyennes mensuelles varient entre 27 et 31 ${ }^{\circ} \mathrm{C}$ et l'humidité relative de l'air fluctue entre $65 \%$ de janvier à mars et $95 \%$ de juin à juillet. Ces élevages qui sont de type traditionnel et semi amélioré ont été sélectionnés d'avance sur des bases bien définies : l'habitat, le mode d'alimentation et le suivi sanitaire.

\section{Animaux et dispositif expérimental Matériel animal}

Cette étude a été réalisée en milieu réel sur deux lots de truies gestantes de race locale. Chaque lot était composé de truies en $2^{\mathrm{e}}$ ou en $3^{\mathrm{e}}$ gestation. Le lot expérimental a été composé de 30 truies et le lot témoin de 36, élevées en élevage traditionnel classique. Dans chaque site, se trouvaient respectivement ces deux lots. A Togba, Ouèdo et Hêvié, chaque lot était composé de 8 truies. A Zinvié, le lot expérimental était composé de 6 truies et lot témoin, 12 truies. Au total, la collecte des données a été réalisée dans 8 exploitations dont 4 pour les lots expérimentaux et 4 pour les témoins. Dans chaque localité, deux exploitations ont été retenues dont une a été destinée au lot expérimental et l'autre au lot témoin.

\section{Habitats}

Au cours de l'expérience, les porcs ont été logés dans de petits enclos de forme rectangulaire confectionnés en bois ou en terre battue. Quelques branchages et/ou feuilles de tôles ont été disposés en guise de toiture et ont servi de protection contre l'insolation et les intempéries. Les porcheries étaient situées à quelques dizaines de mètres des habitations, et il n'a pas été rare de trouver plusieurs porcheries regroupées au même endroit. Ce type de porcherie a été utilisé pour les animaux témoins. L'entretien de l'habitat est occasionnel. La porcherie utilisée pour les animaux du lot expérimental a été de type semi ouvert avec des murets de 1 mètre de haut, faits de briques en ciment ou en terre rouge surélevé. Chaque loge est munie d'un abreuvoir et d'une mangeoire. La toiture est en tôles ou en chaumes et le sol est cimenté et l'entretien de l'habitat est quotidien. Les truies étaient logées individuellement dans le lot expérimental et en promiscuité dans les élevages traditionnels.

\section{Suivi sanitaire}

Au début de l'expérimentation, chaque truie du lot expérimental a été déparasitée contre les parasitoses gastro-intestinales avec l'Alfamec $^{\circledR}$ (ivermectine). A l'âge de 7 jours, les porcelets ont reçu le fer pour éviter la crise de trois semaines. A l'âge de 6 semaines, les porcelets ont été déparasités contre les parasitoses gastro-intestinales. Les animaux malades ont été systématiquement traités sur la base des signes cliniques observés. Le schéma de chimioprévention et de chimiothérapie ainsi que les produits utilisés et leurs principes actifs sont présentés au tableau 1.

Pour le lot témoin, aucun programme de suivi sanitaire n'a été observé au cours de l'expérimentation.
Alimentation
Un mois avant la mise bas, chaque truie gestante du lot expérimental a reçu un complément de ration pour couvrir les dépenses énergétiques liées au développement des fotus dans le dernier tiers de la gestation.

Tableau 1: Chimioprévention et chimiothérapie appliquées aux animaux du lot expérimental.

\begin{tabular}{llll}
\hline Produits & \multicolumn{1}{c}{ Principe actif } & \multicolumn{1}{c}{ Traitement } & \multicolumn{1}{c}{ Période } \\
\hline Oxytétracycline 5\% & Oxytétracycline & Maladies sensibles & En cas de maladie \\
\hline Alfamec ${ }^{\circledR}$ & Ivermectine & $\begin{array}{l}\text { Parasites externes } \\
\text { et internes }\end{array}$ & $\begin{array}{l}\text { Début de gestation et à la 6 } \\
\text { seme }\end{array}$ \\
\hline Fercobsang chez les porcelets
\end{tabular}


Les animaux passaient la nuit dans la porcherie et le matin à dix heures, ils étaient libérés après avoir consommé leur complément alimentaire. La quantité d'aliment servie a été de $500 \mathrm{~g}$ par truie et par jour pendant la gestation. Les truies allaitantes ont reçu un complément d'aliment proportionnément au nombre de porcelets : en moyenne, $1 \mathrm{~kg}$ pour une portée de moins 5 porcelets et $1,5 \mathrm{~kg}$ pour les portées supérieures à 5 porcelets. L'alimentation des truies a été ainsi complémentée du dernier mois de la gestation à l'âge de 60 jours. La composition centésimale de l'aliment des truies du lot expérimental est donnée au tableau 2. L'énergie métabolisable de cet aliment a été de $2600 \mathrm{kcal} / \mathrm{kg}$ d'aliment et contenaient $17,51 \%$ de MAT. Dans le lot témoin, les animaux ont été élevés en divagation totale le jour et passaient la nuit dans leur habitat. Dans ce type d'élevage, l'apport de compléments est rare.

\section{Collecte des données}

Pour chacun des deux lots, le nombre de porcelets nés totaux, le nombre de porcelets nés vivants, le nombre de morts nés, le nombre de morts naissance-sevrage et le poids individuel à la naisssance ont été enregistrés. Les porcelets ont été pesés dans les 72 heures après la mise bas, en raison de l'éloignement des sites d'étude et des difficultés de communication. De façon régulière, les animaux ont été pesés tous les 08 jours, jusqu'à l'âge de 56 jours.

\section{Analyse statistique}

Les données de poids et de reproduction (taille de la portée, nombre de morts nés, nombre de nés vivants, nombre de morts avant le sevrage) ont été enregistrées sur une base de données. Les taux de mortinatalité et de mortalité naissance sevrage ont été calculés. Il en a été de même pour les gains moyens quotidiens des quatre premières semaines (GMQ04), des quatre dernières semaines (GMQ48) et de la naissance au sevrage (GMQ08). Les analyses ont été réalisées à partir du logiciel SAS (1989) et plusieurs procécédures ont été utilisées.

Pour le poids à âge-type et les gains moyens quotidiens, les effets fixes considérés dans le modèle d'analyse ont été le lot et le sexe. Le rang de mise bas, la localité et l'interaction entre le lot et le sexe, n'ont pas été significatifs et par conséquent, n'ont pas été pris en compte lors de l'analyse des données. Des comparaisons ont été faites entre les mâles et entre les femelles des deux lots (expérimental et témoin). La procédure des modèles linéaires généralisés a été utilisée pour déterminer le seuil de signification de chaque effet du modèle à travers le test de $\mathrm{F}$ et les comparaisons entre les moyennes ont été réalisées deux à deux en utilisant le test de t.

En ce qui concerne les paramètres de reproduction, la taille de la portée, le nombre de morts nés, le nombre de nés vivants, le nombre de sevrés, l'âge au sevrage, le poids de la portée à la mise bas et au sevrage, ont été calculés et comparés par lots. Le test de $\mathrm{t}$ a été utilisé pour la comparaison des moyennes. Les taux de mortinatalité et de mortalité naissance-sevrage ont été comparés en utilisant le test de Chi-carré à partir de la procédure Proc freq du SAS (1989).

Tableau 2 : Composition centésimale de l'aliment des truies du lot expérimental.

\begin{tabular}{lc}
\hline Matière première & Composition centésimale (\%) \\
\hline Son de maïs & 50,5 \\
Tourteau de palmiste & 33 \\
Tourteau de soja & 10 \\
Tourteau de coton & 5 \\
Coquille d'huître & 1 \\
Sel de cuisine & 0,4 \\
Phosphate bicalcique & 0,1 \\
\hline
\end{tabular}


RESULTATS

\section{Productivité numérique}

Le tableau 3 présente les paramètres de reproductions des truies des lots expérimental et témoin. Dans le lot témoin, 36 truies locales gestantes ont été enregistrées et toutes ces truies ont mis bas au total 192 porcelets locaux tandis que celui expérimental constitué de 30 truies locales gestantes a donné un total de 352 porcelets locaux nés et toutes ces truies ont également mis bas. La moyenne de la taille de la portée des truies locales du lot témoin $(5,33 \pm 2,06)$ a été plus faible $(\mathrm{P}<0,001)$ que celle des truies expérimentales $(8,8 \pm 3,21)$. Parmi les porcelets nés par portée, il a été observé plus de nés vivants dans le lot expérimental $(8,3)$ que dans le lot témoin $(4,89)$. De même, le poids de la portée a été plus important $(\mathrm{P}<0,05)$ chez le lot expérimental comparativement au lot témoin à la mise bas. Le nombre de porcelets sevrés dans le lot expérimental $(5,6)$ a été plus important $(\mathrm{P}<0,01)$ que celui obtenu dans le lot témoin $(3,44)$. Par rapport au lot témoin, les porcelets du lot expérimental ont été précocement sevrés $(\mathrm{P}<0,01)$ avec un poids de portée significativement plus élevé $(\mathrm{P}<0,001)$. Le taux de mortinatalité des porcelets du lot témoin $(12,5 \%)$ a été le double de celui obtenu chez les porcelets du lot expérimental $(5,68 \%)$. Le taux de mortalité naissance sevrage a été de $32,53 \%$ chez les porcelets du lot expérimental et $29,54 \%$ chez les porcelets du lot témoin mais aucune différence significative n'a été observée entre ces deux taux.

Effet du mode d'élevage sur les performances pondérales

A la naissance, le poids des porcelets locaux du lot expérimental $(650,27 \mathrm{~g})$ a été proche de celui du lot témoin $(657,87 \mathrm{~g})$ et aucune différence significative n'a été observée entre le poids à la naissance des porcelets des deux lots $(P>0,05)$. De la naissance au sevrage, le poids à âge type des porcelets expérimentaux a été significativement plus élevé par rapport à ceux des témoins. Au sevrage, l'écart de poids entre les porcelets témoins et ceux du lot expérimental a été de $544 \mathrm{~g}$ en faveur du lot expérimental. Le poids à âge-type des porcelets des deux lots est donné au tableau 4. Par ailleurs, pendant les quatre premières semaines, le gain moyen quotidien des porcelets du lot expérimental $(55,05 \pm 1,99$ $\mathrm{g} / \mathrm{j}$ ) a été significativement plus élevé que celui du lot témoin $(34,20 \mathrm{~g} / \mathrm{j})$. La même tendance a été observée de la $4 \mathrm{e}$ à la $8 \mathrm{e}$ semaine avec des gains moyens quotidiens de $46,79 \mathrm{~g} / \mathrm{j}$ et $37,77 \mathrm{~g} / \mathrm{j}$, respectivement pour les porcelets expérimentaux et témoins. Pendant toute la durée de l'expérience, les porcelets du lot expérimental ont eu une meilleure croissance $(\mathrm{P}<0,001)$ par rapport à ceux du lot témoin (Tableau 4).

Tableau 3: Variabilité de la productivité numérique du porc local du Bénin.

\begin{tabular}{lccc}
\hline Variables & $\begin{array}{c}\text { Lot Expérimental } \\
(\text { moyenne } \pm \text { DS })\end{array}$ & $\begin{array}{c}\text { Lot Témoin } \\
(\text { moyenne } \pm \text { DS) }\end{array}$ & $\begin{array}{c}\text { Test de } \\
\text { signification }\end{array}$ \\
\hline Taille de la portée & $8,8 \pm 3,21$ & $5,33 \pm 2,06$ & $* * *$ \\
Mort-nés & $0,5 \pm 1,05$ & $0,67 \pm 0,97$ & $\mathrm{NS}$ \\
Nés vivants & $8,3 \pm 3,25$ & $4,89 \pm 1,71$ & $* * *$ \\
Nombre de sevrés & $5,6 \pm 2,56$ & $3,44 \pm 1,46$ & $* *$ \\
Age au sevrage (jour) & $50,3 \pm 2,39$ & $52,67 \pm 2,0$ & $* *$ \\
Poids de la portée à la mise bas $(\mathrm{g})$ & $5130 \pm 2079,88$ & $3681,67 \pm 1370,81$ & $*$ \\
Poids de la portée au sevrage $(\mathrm{g})$ & $20339 \pm 10793,34$ & $9638,89 \pm 5967,25$ & $* * *$ \\
\hline \multicolumn{2}{r}{$: \mathrm{P}<0,05 ; * *: \mathrm{P}<0,01 ; * * *: \mathrm{P}<0,001 ; \mathrm{NS}: \mathrm{P}>0,05 ; \mathrm{DS}:$ déviation standard. }
\end{tabular}


G. B. KOUTINHOUIN et al. / Int. J. Biol. Chem. Sci. 3(4): 819-829, 2009

Tableau 4: Moyennes moindres carrés des performances de croissance des porcelets par lot et par sexe.

\begin{tabular}{|c|c|c|c|c|c|c|c|}
\hline \multirow[b]{2}{*}{ Variables } & \multicolumn{2}{|c|}{ Lot } & \multicolumn{2}{|c|}{ Sexe } & \multirow{2}{*}{$\begin{array}{c}\text { Test de } \\
\text { signification } \\
\text { Lot }\end{array}$} & \multirow{2}{*}{$\begin{array}{c}\text { Test de } \\
\text { signification } \\
\text { Sexe }\end{array}$} & \multirow[t]{2}{*}{$\mathrm{R}^{2}$} \\
\hline & $\begin{array}{c}\text { Expérimental } \\
\text { (Moyenne } \pm \text { ES) }\end{array}$ & $\begin{array}{c}\text { Témoin } \\
\text { (Moyenne } \pm \text { ES) }\end{array}$ & $\begin{array}{c}\text { Femelle } \\
\text { (Moyenne } \pm \text { ES) }\end{array}$ & $\begin{array}{c}\text { Mâle } \\
(\text { Moyenne } \pm \mathrm{ES})\end{array}$ & & & \\
\hline $\mathrm{P} 0$ (g) & $650,27 \pm 13,02$ & $657,87 \pm 16,46$ & $647,15 \pm 15,64$ & $660,98 \pm 13,99$ & NS & NS & 0,02 \\
\hline P1 (g) & $1112,36 \pm 28,9$ & $960,14 \pm 36,95$ & $1023 \pm 35$ & $1049,5 \pm 31,23$ & $* *$ & NS & 0,07 \\
\hline P2 (g) & $1498,29 \pm 47,14$ & $1293,75 \pm 64,56$ & $1375,3 \pm 58,93$ & $1416,8 \pm 54,01$ & $*$ & NS & 0,06 \\
\hline P5 (g) & $2496,97 \pm 72,48$ & $1848,86 \pm 95,22$ & $2090,8 \pm 86,69$ & $2255,1 \pm 82,49$ & $* * *$ & NS & 0,15 \\
\hline P6 (g) & $2895,74 \pm 80,68$ & $2176,1 \pm 105,83$ & $2416,1 \pm 95,68$ & $2646,7 \pm 92,5$ & $* * *$ & NS & 0,16 \\
\hline P7 (g) & $3241,97 \pm 112,64$ & $2509,08 \pm 126,63$ & $2815,8 \pm 121,08$ & $2935,3 \pm 118,58$ & $* * *$ & NS & 0,12 \\
\hline P8 (g) & $3463,76 \pm 117,17$ & $2919,35 \pm 129,84$ & $3142,4 \pm 129,84$ & $3240,7 \pm 117,17$ & $* *$ & NS & 0,08 \\
\hline
\end{tabular}

*: $\mathrm{P}<0,05 ; * *: \mathrm{P}<0,01 ; * * *: \mathrm{P}<0,001 ; \mathrm{NS}: \mathrm{P}>0,05 ; \mathrm{ES}$ : Erreur Standard ; Pi : Poids à la ième semaine; GMQ04: gain moyen quotidien entre la mise bas et la $4^{\text {eme }}$
semaine; GMQ48: gain moyen quotidien entre la $4^{\text {eme }}$ et la $8^{\text {ème }}$ semaine; GMQ08: gain moyen quotidien entre la mise bas et la $8^{\text {eme }}$ semaine. 
Effet du sexe sur les performances pondérales

En considérant l'ensemble des animaux, les poids à âge-type et les gains moyens quotidiens des mâles et des femelles ont été identiques de la naissance au sevrage (tableau 4). Le poids à la naissance des mâles a été de $661 \mathrm{~g}$ et celui des femelles, 647,15 g. $\mathrm{Au}$ sevrage, les mâles pesaient $3241 \mathrm{~g}$ et les femelles $3142 \mathrm{~g}$.

L'interaction entre lot et sexe n'a pas été significative pour les poids à âge-type et les gains moyens quotidiens. Cependant, en comparant les performances pondérales des femelles des deux lots, il apparaît que les poids étaient identiques à la mise bas et pendant les deux premières semaines de croissance. De la $3^{\mathrm{e}}$ semaine à la fin de l'expérimentation, les femelles du lot expérimental ont plus pesé que celles du lot témoin $(\mathrm{P}<0,001)$. Le même résultat a été également obtenu entre les mâles des deux lots. L'évolution du poids à âge-type de la naissance au sevrage des porcelets par lot est donnée pour les femelles et les mâles respectivement dans les figures 2 et 3 .

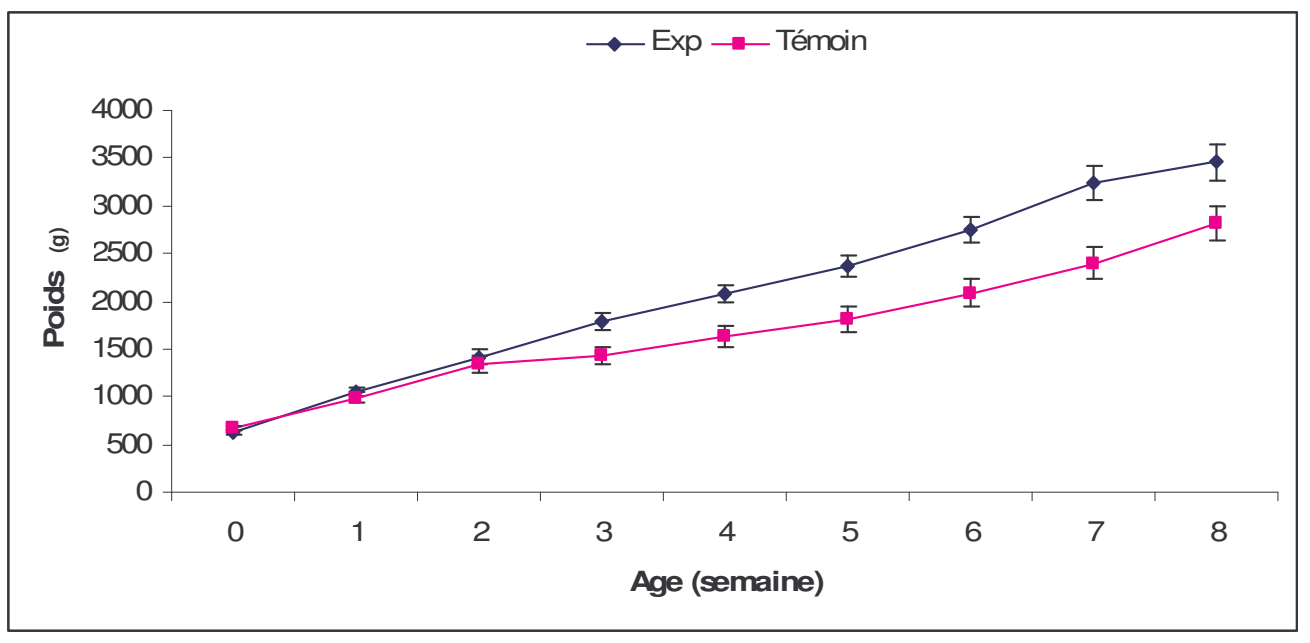

Figure 2 : Evolution pondérale des femelles des lots expérimental et témoin de la naissance au sevrage.

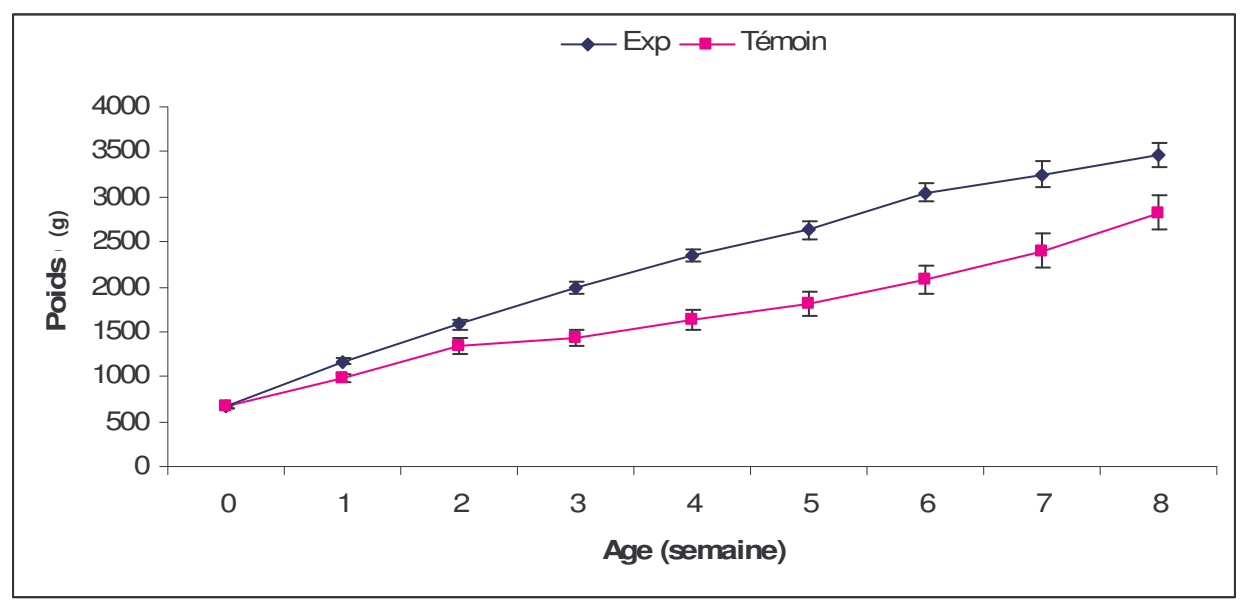

Figure 3 : Evolution pondérale des mâles des lots expérimental et témoin de la naissance au sevrage. 


\section{DISCUSSION \\ Productivité numérique \\ Taille de la portée}

La taille moyenne de la portée du lot témoin a été de 5,33 porcelets. Cette moyenne est faible par rapport à celle des truies locales du lot expérimental qui a été de 8,8 porcelets. La différence significative observée au cours de notre étude s'explique par la réduction de la mortalité embryonnaire sous l'effet du complément alimentaire. Dans les élevages traditionnels où les animaux sont en divagation et peu suivis sur le plan sanitaire, le nombre moyen de porcelets nés vivants est de 4,74 (Attodjinou et Dotcho, 2006). Ces observations vont dans le même sens que celles de Quiniou et Maupertuis (2007) qui ont rapporté que l'augmentation de la ration en fin de gestation est associée à une plus grande facilité de mise bas et une meilleure vitalité néo-natale des porcelets. A trois jours de vie, les porcelets nés en plein air sont également plus homogènes, ce qui se traduit par une taille de portée plus importante au sevrage. La taille de la portée obtenue au cours de la présente étude a été meilleure à celles obtenues à la Faculté des Sciences Agronomiques de l'Université d'AbomeyCalavi par d'Orgeval (1997) et à l'Ecole Polytechnique d'Abomey-Calavi (Yacoubou, 2007 ; Youssao et al., 2009). Un récapitulatif de la taille de la portée des porcs locaux du Bénin et des pays voisins comme le Nigéria et le Cameroun est donné au tableau 5.

\section{Mortalité}

Le taux de mortinatalité des porcelets locaux du lot témoin a été le double de celui du lot expérimental. Ce taux élevé chez les témoins pourrait s'expliquer par la sousalimentation, la température ambiante et la taille de la portée (Holnes, 1991). Les truies du lot expérimental bénéficient en effet d'un environnement un peu plus biosécurisé (habitat et hygiène) comparativement à ceux du lot témoin.

En ce qui concerne les taux de mortalité naissance-sevrage, le lot témoin $(29,54 \%)$ a une mortalité relativement proche de celui du lot expérimental (32,53\%). L'alimentation des mères et le suivi sanitaire des truies après la mise bas n'ont eu aucun effet sur le taux de mortalité naissancesevrage. Toutefois, dans les deux lots, le taux de mortalité naissance-sevrage est très important pour un élevage lucratif. Des taux similaires ont été obtenus dans d'autres études (Nonfon et al., 2000 ; Agbokounou, 2001 ). Selon Attodjinou et Dotcho (2006), dans les élevages traditionnels au Sud du Bénin, $21,74 \%$ de cas de mortalité ont été observées de la naissance au sevrage. Un taux de mortalité de $29,12 \%$ a été obtenu par Nonfon et al. (2000) lors de l'étude des paramètres de production du porc local au Centre de Recherche et de Développement du Porc Local de 1989 à 1994 à la Faculté des Sciences Agronomiques de l'Université d'Abomey-Calavi. Des taux de mortalité naissance-sevrage variant de 15 à $19 \%$ chez les porcs locaux ont été rapportés par d'Orgeval (2007) alors que dans la présente étude, de la naissance au sevrage, ce taux a varié de 29 à $32 \%$.

De manière générale, les taux de mortinatalité et de mortalité des porcs locaux sont globalement élevés et une étude de morbidité et de mortalité serait nécessaire pour améliorer la viabilité des animaux. Les différents taux de mortalités de la naissance au sevrage sont présentés au tableau 4.

\section{Performances pondérales}

A la naissance, les poids des porcelets des lots témoin et expérimental ont été respectivement de $657,87 \mathrm{~g}$ et $650,27 \mathrm{~g}$ et ne diffèrent pas significativement. Toutefois, eu égard à la portée plus large des truies du lot expérimental, les porcelets du lot expérimental ont des performances pondérales individuelles relatives plus importantes que celle du lot témoin. Les résultats obtenus dans la présente étude montrent qu'il n'y a pas une différence importante entre le poids à la naissance des races locales en fonction du mode d'élevage traditionnel. A la ferme de Kpinnou, le poids moyen des porcelets a été de 510g (Bonou, 2006) et est inférieur aux résultats de notre étude. De la naissance au sevrage, le poids à âge-type des porcelets du lot expérimental a été significativement plus élevé par rapport à ceux du lot témoin. Cette différence significative pourrait s'expliquer par une meilleure production laitière des femelles du lot expérimental sous l'effet du complément alimentaire. Les poids moyens de la portée du lot expérimental et du lot témoin 
Tableau 5: Performances de reproduction des porcs locaux.

\begin{tabular}{|c|c|c|c|c|c|c|}
\hline Lieu & $\begin{array}{l}\text { Nombre de } \\
\text { portées observées }\end{array}$ & $\begin{array}{l}\text { Taille de la portée } \\
\text { à la naissance }\end{array}$ & $\begin{array}{c}\text { Poids à la } \\
\text { naissance (kg) }\end{array}$ & $\begin{array}{c}\text { Taux de mortalité } \\
\text { au sevrage }(\%)\end{array}$ & $\begin{array}{c}\text { GMQ de la naissance } \\
\text { au sevrage }(\mathrm{g} / \mathbf{j})\end{array}$ & Source \\
\hline Bénin (FSA) & - & 7,3 & 0,92 & 19,1 & 103 & d'Orgeval(1997) \\
\hline Bénin (EPAC) & - & - & 0,51 & - & - & Adjakpa (2005) \\
\hline Bénin (EPAC) & 23 & 7,67 & 0,53 & 38,89 & 52 & Yacoubou (2007) \\
\hline Bénin (élevage traditionnel) & 22 & 5,74 & $0,81^{*}$ & 21,74 & - & $\begin{array}{l}\text { Attodjinou et } \\
\text { Dotcho (2006) }\end{array}$ \\
\hline Bénin (FEK) & 442 & 6,31 & 0,51 & 9,05 & - & Bonou (2006) \\
\hline Bénin (EPAC) & 122 & 7,25 & 0,53 & 12,6 & 70 & Youssao et al. (2009) \\
\hline Nigeria & - & 6,7 & 0,91 & 15,8 & 102 & d'Orgeval (1997) \\
\hline Cameroun & - & 7,8 & 1,15 & 22 & 106 & d'Orgeval (1997) \\
\hline
\end{tabular}


ont été respectivement $5130 \mathrm{~g}$ et $3681,67 \mathrm{~g}$. Ces résultats confirment ceux obtenus par Quiniou et Maupertuis (2007).

En général, les performances de croissance ont été plus bonnes dans le lot expérimental que dans le lot témoin et cette différence pourrait s'expliquer par l'apport du complément alimentaire des mères allaitantes. Malgré cette différence, le gain moyen quotidien obtenu dans le lot expérimental de la présente étude est inférieur à ceux rapportés dans de nombreuses expérimentations. Lors de l'étude des besoins énergétiques et protéiques du porc local béninois en phase de démarrage-croissance (4 à $10 \mathrm{~kg}$ de poids vif), Agbokounou (2001) a obtenu des GMQ de $131,105,156$ et $137 \mathrm{~g} / \mathrm{j}$ à partir des rations d'énergie respectives de 13,9, 14, 15,2 et 15,2 $\mathrm{MJ} / \mathrm{kg} \quad \mathrm{d}$ 'aliment, correspondant respectivement à une teneur en MAT de 17,5, $18,8,16,2$ et $18,3 \%$. Ces gains sont comparables à ceux obtenus par Youssao et al. (2004a) dans les porcs locaux. Des gains moyens quotidiens (non significativement différents) de $200 \mathrm{~g} / \mathrm{j}$ et $184 \mathrm{~g} / \mathrm{j}$ ont été respectivement obtenus pour la ration hautement énergétique $(13,49 \mathrm{MJ} / \mathrm{kg}$ de $\mathrm{MS}$ et $19,01 \%$ de protéines brutes) et la ration faiblement énergétique $(12,24 \mathrm{MJ} / \mathrm{kg}$ de $\mathrm{MS}$ et $19,81 \%$ de protéines brutes) lors de l'estimation des besoins énergétiques du porc local du Bénin en croissance entre 2 et $22 \mathrm{~kg}$ de poids vif (Codjo, 2003). La race porcine locale élevée dans des conditions d'élevage traditionnel, a un gain de $54 \mathrm{~g} / \mathrm{j}$ (Youssao et al., 2004b). Des gains comparables (66 g/j) ont été obtenus par d'Orgeval (1997) sur des porcs de race locale de la naissance à un poids moyen de $10 \mathrm{~kg}$ dans les élevages traditionnels. Tenant compte de ces résultats, l'amélioration des conditions d'élevage en général et l'alimentation en particulier permettront aux races autochtones de mieux exprimer leur potentiel génétique.

\section{Conclusion}

L'étude de l'effet du mode d'élevage sur la prolificité des truies de race locale et la viabilité de leurs porcelets, de la naissance au sevrage, a été réalisée dans le Département de l'Atlantique et il en ressort que l'amélioration du mode d'élevage des truies gestantes augmente la taille et le poids de la portée et réduit le taux de mortinatalité. Au sevrage, l'apport de compléments d'aliment à la truie allaitante améliore le poids au sevrage des porcelets. Toutefois, l'apport de complément n'a aucun effet sur le taux de mortalité naissance-sevrage. A l'issue de cette étude, il serait souhaitable d'étudier les facteurs conditionnant la productivité numérique des truies locales et de poursuivre des travaux sur l'alimentation des truies gestantes et allaitantes pour améliorer la productivité numérique et la viabilité des porcelets, de la naissance au sevrage.

\section{REMERCIEMENTS}

Les auteurs remercient APRA pour son appui à la réalisation de ce travail.

\section{REFERENCES BIBLIOGRAPHIQUES}

Agbokounou AM. 2001. Etude des besoins énergétiques et protéiques du porc local béninois en phase de démarragecroissance. Mémoire de D.E.A. en Zootechnie, Faculté universitaire des sciences agronomiques de Gembloux, Belgique, 92.

Attodjinou FTR, Dotcho CDG. 2006. Caractéristiques de l'élevage des porcs locaux dans les élevages périurbains de Cotonou. Mémoire de fin de formation, Sékou, 72.

Bonou D. 2006. Etude comparative de quelques performances zootechniques des porcs locaux et métis issu du croisement entre Large White et Landrace. Mémoire d'Ingénieur des Travaux, Université d'Abomey-Calavi, Bénin, 63.

Codjo AB. 2003. Estimation des besoins énergétiques du porc local du Bénin en croissance entre 2 et $22 \mathrm{~kg}$ de poids vif. Tropicultura, 21(2): 56-60.

D’Orgeval R. 1997. Le développement de l'élevage porcin en Afrique: l'analyse des systèmes d'élevage du porc local africain au Sud Bénin. Thèse de Doctorat de l'Institut National Agronomique, Paris Grignon, 273.

FAO, 2005. Banques de données, FAOSTAT: Agriculture. Consulté le 27 février 2005 à l'adresse: http://apps.fao.org/page/ collections?subset=agriculture $\&$ language $=\mathrm{FR}$. 
Holnes HD. 1991. Le Porc, CTA, édition; 217.

Honkpehedji HV. 2005. Influence du système délevage sur les performances de croissance du porc de race local du Bénin à l'engrais. Mémoire d'Ingénieur des Travaux, Université d'Abomey-Calavi, Bénin, 51.

Nonfon WR, Deka E, Adegbidji A, Codjo B, Chrysostome C. 2000. Amélioration de la productivité du porc local au sud Bénin. Rapport technique final, Université nationale du Bénin/FSA, Cotonou, Bénin, p.174 Quiniou N, Maupertuis F. 2007. Il faut augmenter la ration allouée à la truie hyperprolifique pendant les dernières semaines de gestation. Consulté le 27 avril 2008 à l'adresse: http://www.itp.asso.fr/lirfor/techpor/intro/ ali/alitruie.htm\#getyperra .

SAS. 1989. SAS/STAT. User's Guide (Ressource Electronique) (4 ${ }^{\text {ème }}$ édn).
Version 8 - SAS. Inst. Inc. : Cary, NewYork.

Youssao AKI, Mourot J, Gbangboche AB, Adehan R, Akoutey A, Edenakpo A. 2004a. Influence du régime alimentaire sur les performances de croissance et les caractéristiques de la carcasse du porc de race locale du Bénin. RASPA, 2(1): 3136.

Youssao AKI, Mourot J, Edenakpo A. 2004b. Effet du mode d'élevage sur les caractéristiques de la carcasse et de la viande du porc local du Bénin. $10^{\mathrm{e}}$ Journées des Sciences du Muscle et la Technologie de la Viande (JSMTV): 153-154.

Youssao AKI, Koutinhouin GB, Kpodekon TM, Bonou AG, Adjakpa A, Ahounou GS, Mourot J, 2009. Performances zootechniques et aptitudes bouchères des porcs locaux au Sud du Bénin. Bull. Anim. Hlth. Prod. Afr., 57: 73-87. 